\title{
Candida lusitaniae EN UN PACIENTE PEDIATRICO INMUNOCOMPROMETIDO: EXITO TERAPEUTICO DEL VORICONAZOL
}

(Candida lusitaniae in an immunocompromised patient: Therapeutic success of Voriconazole)

\author{
Rosa Runco $^{1,2}$ \& Raquel Salim ${ }^{1}$ \\ 1. Cátedra de Micología. Instituto de Microbiología «Dr. Luis C. Verna». \\ Facultad de Bioquímica, Química y Farmacia - Universidad Nacional \\ de Tucumán. Ayacucho 491. (4000) San Miguel de Tucumán. R. Argentina. \\ 2. Laboratorio de Micología del Hospital del Niño Jesús. Pasaje Hungría 750. \\ (4000) San Miguel de Tucumán. R. Argentina. \\ e-mail: rqsalim@unt.edu.ar
}

Palabras clave: Candida lusitaniae, paciente pediátrico, Voriconazol

Key words: Candida lusitaniae, paediatric patient, Voriconazole

\section{RESUMEN}

Candida lusitaniae es reconocida como un patógeno emergente, ocasionalmente responsable de graves infecciones nosocomiales en pacientes con compromiso inmune. Esta levadura plantea dos desafíos importantes; la posibilidad de una identificación errónea de la especie y su resistencia primaria o adquirida a la Anfotericina B. El presente trabajo informa el aislamiento en cultivos puros de $\boldsymbol{C}$.lusitaniae desde el líquido cefalorraquídeo de un paciente pediátrico inmunocomprometido, de 5 meses de edad, hospitalizado por hidrocefalia posthemorrágia neonatal valvulada. El paciente fue tratado inicialmente con Anfotericina B, substituida posteriormente por Voriconazol. Se destaca el éxito terapéutico de éste último en la dosis usada en este caso. Luego de un prolongado período de hospitalización el paciente fue dado de alta. Consideramos que para entender la importancia clínica de esta levadura, es necesario investigaciones más completas tanto de su epidemiología como de los mecanismos de resistencia a los antifúngicos y de su frecuencia, tanto in vivo como in vitro, condiciones útiles para entender algunas de las razones de la emergencia de C. Iusitaniae en clínica.

\section{INTRODUCCION}

A pesar de que se han identificado actualmente unas 200 especies de Candida, sólo unas pocas son habitualmente causantes de enfermedades humanas. Durante las dos décadas pasadas han sucedido cambios
ABSTRACT

Candida lusitaniae is known to be an emerging pathogenous which is occasionally responsible for serious nosocomial infections in immunocompromised patients. This yeast involves two significant challenges: the possibility of a wrong identification of the species and its primary or acquired resistance to Anfotericina B. The present paper reports the isolation of C.lusitaniae in pure cultures taken from a 5-month aged immunocompromised pediatric patient who had been diagnosed with a neonatal post hemorrhagic valvular hydrocephalus. The patient was firstly treated with Anfotericena B and later on with Voriconazole. The therapeutic success of the latter medication as used in this case is pointed out. After being a long time in the hospital, the patient was sent home. In order to understand the clinical significance of this yeast, we think it is necessary to carry out further research work both in its epidemiology and in its antifungal drug resistance mechanisms together with its frequency in situ and in vitro,being the above requirements useful to recognize the reasons for. Clusitaniae emergence in the clinic.

en la distribución de las especies de este género que causan infección hospitalaria. Numerosos informes han documentado la aparición de especies distintas a $\boldsymbol{C}$. albicans como C. glabrata C. krusei, C. guilliermondii, C. dubliniensis, y C. lusitaniae entre otras, responsables de graves y aun fatales infecciones en el hombre. Este 
hecho puede estar relacionado con el uso creciente de antifúngicos, tanto en profilaxis como en tratamientos empíricos (2, 6,9,10,11,15,17,19,20,32).

C. Iusitaniae van Uden \& Do Carmo-Sousa, se encuentra colonizando el tracto respiratorio, gastrointestinal y urinario humano. Se ha encontrado también en el tracto digestivo de animales, leche de vacas con mastitis, granos de maíz, cáscara de citrus y jugos de frutas (14).

Fue descrita originalmente por Langeron y Talice en 1954 como Candida parapsilosis var. obtusa y fue aislada por primera vez en 1959 por Van Uden y do CarmoSousa como microorganismo de la microbiota intestinal de animales de sangre caliente (14) y su forma teleomorfa se denomina Clavispora lusitaniae Rodríguez de Miranda (Ascomycota, Hemiascomycetes, Saccharomycetales: Metschnikowiacae). Después, en 1979, Papagianis (21) \& Holschu (11), describieron los primeros casos de infección humana oportunista a la vez que demostraron su capacidad para desarrollar resistencia a la anfotericina B en el curso del tratamiento. Posteriormente otros autores publicaron los primeros casos de aislamiento de la levadura en sangre y casos de infección nosocomial, algunos por mecanismo de transmisión exógeno, y en otras por infección cruzada $(2,5,9,31,32,33)$.

C. Iusitaniae se comporta como una levadura patógena oportunista, por lo tanto, comparte con otras especies del género los factores de riesgo para la colonización, tales como: neutropenia profunda y prolongada, implantación de material protésico, uso de catéteres endovenosos, prematuridad, corticoterapia, quimioterapia y el uso prolongado de antimicrobianos de amplio espectro, y en general cualquier tipo de inmunosupresión (2,8,9,19, 26,32,33).

Si bien se trata de un microrganismo con baja incidencia de infección en personas inmunocompetentes, actualmente es reconocida como un patógeno nosocomial emergente en pacientes graves e inmunocomprometidos, incluyendo septicemia, meningitis, pielonefritis, osteomielitis e infecciones del tracto respiratorio, urinario y gastrointestinal, en general de mal pronóstico $(8,9,32,33)$.

Desde el punto de vista clínico la resistencia inicial o el desarrollo rápido de resistencia a anfotericina B constituye la característica más importante de $\boldsymbol{C}$. lusitaniae. Muchos autores consideran que esta resistencia es adquirida aunque también se ha documentado cepas con resistencia intrínseca $(1,4,7,8,15,17,24,34)$.

La anfotericina B sigue siendo la droga de elección para el tratamiento de muchas infecciones fúngicas sistémicas, inclusive las infecciones asociadas con $\boldsymbol{C}$. lusitaniae, por lo que la detección de esta resistencia es esencial para el monitoreo efectivo del tratamiento antifúngico. Por otra parte se han descrito casos de fracasos terapéuticos asociados no sólo con la resistencia de esta levadura a la anfotericina B sino también a los azoles tanto en pacientes inmunocomprometidos como inmunocompetentes $(2,34)$.

Favel et al. (4), estudiaron la susceptibilidad in vitro de 80 aislamientos clínicos de $\boldsymbol{C}$. Iusitaniae frente a cinco agentes antifúngicos sistémicos y encontraron que todos los aislamientos fueron altamente sensibles a Fluconazol y a Voriconazol. Hsue et al. (12), estudiaron la susceptibilidad de 391 aislamientos de Candida spp. frente a seis agentes antifúngicos, obteniendo resultados similares y señalan que el Voriconazol resulta el agente más efectivo contra los aislamientos fúngicos ensayados, incluyendo Fluconazol y cepas no susceptibles a Anfotericina B.

Debido a la baja frecuencia del aislamiento de $\boldsymbol{C}$. lusitaniae en clínica, no se dispone de datos concluyentes sobre su tratamiento antifúngico de elección, sin embargo, frente a la aparición de cepas resistentes a la anfotericina $\mathrm{B}$, el tratamiento recomendado es el Fluconazol a dosis de 6-12 mg/Kg/día o Voriconazol (6 mg/Kg/12 h). Como los nuevos azoles muestran actividad in vitro frente a esta levadura, parece razonable asumir que puedan utilizarse para tratar las infecciones causadas por $\boldsymbol{C}$. lusitaniae $(12,16,17,25)$.

El presente trabajo tiene la finalidad de informar el aislamiento en cultivos puros de C.lusitaniae desde líquido cefalorraquídeo de un paciente pediátrico inmunocomprometido hospitalizado y tratado exitosamente con Voriconazol, además de valorar la importancia médica de esta infección.

\section{CASO CLINICO}

Paciente de 5 meses y 15 días de edad, con antecedentes de hemorragia cerebral neonatal con hidrocefalia que fue valvulada, que ingresa al Hospital del Niño Jesús (Tucumán, Argentina) 45 días después de su alta, por presentar irritabilidad, vómitos, rechazo alimentario y aumento del perímetro cefálico.

Examen físico: paciente afebril, hidratado, buena tolerancia oral, diuresis positiva, peso de $4.350 \mathrm{~g}$, palidez cutáneo-mucosa y reticulado marmóreo generalizado. Mucosa oral congestiva con placa blanquecina en cara dorsal de lengua, además de dermatitis del pañal.

Examen neurológico: paciente vigil, hipo-activo y desconectado, ausencia de seguimiento con la mirada, pupilas isocóricas y reactivas. Fondo de ojos: atrofia de papila bilateral, perímetro cefálico de $45,8 \mathrm{~cm}$.

Abdomen: blando, indoloro, depresible, ruidos hidroaéreos positivos, hernia umbilical reductible e incoercible, cicatriz quirúrgica en flanco derecho. Se asume 
como desnutrido de segundo grado (déficit del 33\%),

Examen cardiovascular y respiratorio: frecuencia respiratoria 52 por min., regular entrada de aire bilateral, roncus y subcrepitantes en ambos campos pulmonares. Frecuencia cardiaca 135 por min, silencios libres, pulsos periféricos presentes, buena perfusión periférica.

La conducta inicial es el control de perímetro cefálico, posición semisentada, bombeo valvular espaciado y control de fontanela que se encuentra abombada y mide $8 \times 7 \mathrm{~cm}$. Al tercer día del ingreso (29.07.04) se realiza el primer drenaje de líquido cefalorraquídeo (LCR) desde el reservorio valvular y se envía al laboratorio para examen físico-químico-citológico y cultivo. Se mantiene conducta expectante. Se indica Nistatina $100.000 \mathrm{U} / \mathrm{kg} /$ día. Resultados: LCR xantocrómico, de aspecto límpido, sin coágulos ni sangre. El análisis químico revela $0,20 \mathrm{mg} / \mathrm{dl}$, de glucosa. En el examen citológico se cuentan 32 células/ $\mathrm{mm}^{3}$ con $60 \%$ de neutrófilos. Se agrega al tratamiento Vancomicina (60 mg/kg/día) durante 10 días y Ceftazidima (100 mg/kg/día) durante 5 días.

Cinco días después (03.08.04) el laboratorio de Micología informa el aislamiento de Candida sp. Se remueve válvula bajo anestesia general y se envían a estudio la prótesis y LCR. Por vía venosa central se inicia tratamiento con Anfotericina B en dosis crecientes cumpliéndose el siguiente esquema: 1er día: $0,2 \mathrm{mg} / \mathrm{kg} /$ día; $2^{\circ}$ día: 0,4 mg/kg/día; 3er día: 0,6 mg/kg/día; 4º 0,8 $\mathrm{mg} / \mathrm{kg} /$ día y $5^{\circ}$ al $12^{\circ}$ días: $1,0 \mathrm{mg} / \mathrm{kg} /$ día.

Tres días posteriores a la remoción valvular (06.08.04) se realiza drenaje ventricular, se extraen 170 $\mathrm{cm}^{3}$ de LCR para examen físico-químico-citológico y cultivos. Resultados: LCR xantocrómico, de aspecto límpido, sin coágulos ni sangre, $0,11 \mathrm{mg} / \mathrm{dl}$, de glucosa, 25 células $/ \mathrm{mm}^{3}$ con predominio de linfocitos. Los cultivos bacteriológicos son negativos.

El 10.08.04 se realiza drenaje transfontanelar de $100 \mathrm{~cm}^{3} \mathrm{de}$ LCR y se envía al laboratorio planteándose nuevo drenaje en 48 horas y eventual consecución de válvula pediátrica de presión media.

El día 14.08.04 el laboratorio de Micología informa que la especie de Candida ailada corresponde a C. lusitaniae, resistente a Anfotericina B. A partir del día 15.08.04 se cambia el esquema antifúngico y se inicia tratamiento con Voriconazol (6 mg/Kg cada 12 hs).

A partir de esa fecha se realiza punción de fontanela anterior y aspiración de LCR cada cuatro días. Desde entonces los cultivos son negativos.

El paciente presenta febrículas, cumplió 22 días con catéter en vena femoral derecha sin presentar signos de infección.

Desde la fecha de internación al 25.08.04 el perímetro cefálico incrementó $2.2 \mathrm{~cm}$ (de 45,8 a $48 \mathrm{~cm}$ ) sin signos de hipertensión endocraneana. El paciente cumplió 10 días de tratamiento con Voriconazol $(6 \mathrm{mg} / \mathrm{Kg}$ cada 12 hs). Los estudios de laboratorio presentaron los siguientes resultados: LCR xantocrómico, aspecto límpido, sin coágulos ni sangre, $0,50 \mathrm{mg} / \mathrm{dl}$ de glucosa y $384 \mathrm{mg} / \mathrm{dl}$ de proteínas, 12 células $/ \mathrm{mm}^{3}$ con predominio de linfocitos, cultivos negativos.

El tratamiento con Voriconazol se mantiene a iguales dosis hasta el 01.09.04 (18 días en total). Durante este período el paciente se mantiene afebril, su peso incrementó 470 g y comenzó a probar tolerancia con papillas.

El día 08.09.04 Infectología informa que el paciente se encuentra en condiciones de entrar a cirugía para colocación de válvula. Los estudios complementarios de laboratorio presentan los siguientes resultados: Hematocrito 29\%, Hemoglobina 9,2 g\%, Glucemia 0,72 $\mathrm{mg} / \mathrm{dl}$, Uremia 0,29 mg/dl, Creatinina 0,78 mg/l, KPTT 35 seg, Eritrosedimentación 20 mm.

Después de una semana de evolución se observa una franca mejoría clínica. Dos meses más tarde el paciente es dado de alta. Actualmente está asintomático y es controlado periódicamente.

\section{ESTUDIO MICROBIOLOGICO}

Todas las muestras de líquido cefalorraquídeo fueron centrifugadas a 3000 r.p.m. durante $10 \mathrm{~min}$. El pellet fue sembrado en Agar Sabouraud-glucosado con penicilina (50 U.I./ml) y estreptomicina $\left(80 \mu \mathrm{g} / \mathrm{ml}\right.$ ) e incubado a $37^{\circ} \mathrm{C}$ durante $48 \mathrm{~h}$.

Para la identificación de las levaduras aisladas se emplearon las pruebas convencionales de formación de tubos germinativos, producción de clamidoconidios, desarrollo en CHROMagar-Candida (Hardy Lab.), Candifast (International Microbio IM) para zimogramas y API-32 C (bioMérieux) para auxonogramas.

C. Iusitaniae fue identificada sobre la base de su incapacidad para producir ureasa y formar tubos germinativos, así como por su patrón de fermentación y de asimilación de azúcares.

Para valorar la sensibilidad a los antifúngicos 'in vitro' se utilizó el método de macrodilución en caldo (documento de referencia M27-A estandarizado por el NCCLS para levaduras) para determinar la concentración inhibitoria mínima. Este método se utiliza muy poco actualmente, pero a veces puede ser útil cuando se requiere un resultado rápido y no se dispone de métodos comerciales.

Se probó la actividad de Anfotericina B (Squibb, N.J.) $(1.600 \mu \mathrm{g} / \mathrm{ml})$, Itraconazol, (Janssen) $(1.600 \mu \mathrm{g} / \mathrm{ml})$, Voriconazol (Pfizer) $(1.600 \mu \mathrm{g} / \mathrm{ml})$ y Fluconazol (Pfizer) $(12.800 \mu \mathrm{g} / \mathrm{ml})$. Estos antifúngicos, en forma de polvo, fueron disueltos convenientemente. Anfotericina B, Itraconazol, y Voriconazol son insolubles en agua por lo 
que se utilizó dimetilsulfóxido (DMSO) para su disolución. El Fluconazol fue disuelto en agua destilada estéril. El medio de cultivo utilizado fue el RPMI 1640 con glutamina sin bicarbonato sódico (Sigma) tamponado con ácido morfolino propano sulfónico (MOPS) 0,164 M (Sigma) a $\mathrm{pH} 7,0$.

La cepa aislada resultó resistente a anfotericina B y sensible a los demás antifúngicos ensayados.

El control se realizó utilizando la cepa testigo de C. lusitaniae ATCC 200951 resistente a anfotericina B.

\section{DISCUSION}

Fue en la década de los años 40, coincidiendo con la introducción de los tratamientos antimicrobianos, cuando se observó un aumento considerable de la incidencia de todo tipo de candidosis, tanto profundas como superficiales (10,13,20). En el período 1952-1992 se publicaron treinta y siete reportes describiendo 1.591 casos de infecciones sistémicas causadas por Candida spp. entre pacientes con cáncer, mostrando que especies distintas a C. albicans resultaron responsables del $46 \%$ de todas las infecciones sistémicas. En particular, se identificó $\boldsymbol{C}$. tropicalis en el 25\% de los casos, C. glabrata (8\%), C. parapsilosis (7\%) y C. krusei (4\%) (2,7).

En las últimas décadas las infecciones por levaduras han alcanzado una gran importancia no sólo por su aelevada frecuencia en pacientes con compromiso inmune o enfermos sometidos a técnicas diagnósticas y terapéuticas invasivas, sino además, al surgimiento de formas clínicas diseminadas y a su alta morbilidad y mortalidad. Paralelamente se ha notado un aumento en la aparición de nuevas especies patógenas, cuyo diagnóstico y tratamiento muchas veces resulta dificultoso $(2,6,8,10,11,19,20)$.

Entre los patógenos emergentes se mencionan: Malassezia furfur, Trichosporon asahii (beigelii), Rhodotorula spp., Pichia anomala. Se han identificado más de 17 especies de Candida distintas a $\boldsymbol{C}$. albicans. Entre ellas: C. glabrata, C. parapsilosis, C. tropicalis, $C$. krusei, C. guilliermondii, C. lusitaniae, C. rugosa, $C$. dubliniensis, C. inconspicua, C. sake, C. olmi, C. norvegensis, $\boldsymbol{C}$. valida y $\boldsymbol{C}$. famata entre otras $(6,20,25)$. Incluso organismos antiguamente considerados contaminantes ambientales o solamente industrialmente importantes tales como C. utilis y C. lipolytica, actualmente están implicadas como agentes de funguemia, onicomicosis y enfermedad sistémica $(2,10,17,20,21)$.

La frecuencia con que se aíslan las distintas especies de Candida varía según el país, el hospital, las unidades del mismo, e incluso el año de estudio (2). Todas pueden causar el mismo tipo de enfermedad, desde una candidosis superficial hasta una enfermedad invasiva, sin embargo, la gravedad y las opciones terapéuticas difieren entre las distintas especies, razón por la cual es tan importante lograr su identificación precoz.

Los factores que influyen en la elevada tasa de mortalidad de las infecciones fúngicas son múltiples, pero entre todos destaca el retraso en la instauración del tratamiento; ya sea por la dificultad de atribuir a un aislamiento el carácter de patógeno en determinadas muestras, o bien por la gravedad de la enfermedad de base que puede enmascarar la participación de la levadura en el proceso $(2,27)$. El pronóstico depende, en gran medida, de la precocidad del diagnóstico y del tratamiento. Excepto en la candidemia, el diagnóstico de certeza de una infección fúngica invasiva suele establecerse con la enfermedad avanzada y ello motiva que en la mayoría de los pacientes el tratamiento se inicie empíricamente, como en el caso que nos ocupa $(6,13)$.

Además, el uso profiláctico de antifúngicos y la generalización de la terapia empírica precoz han incrementado la aparición de especies de Candida no albicans y de otras levaduras patógenas emergentes (13). Este incremento se relacionaría no sólo con la alteración de las defensas del huésped sino con ciertas características de patogenicidad, entre ellas la resistencia a los antifúngicos.

García Martos (7), ha constatado la aparición de resistencia, especialmente a derivados azólicos, no sólo por el uso frecuente de antifúngicos sino también al aumento de las dosis terapéuticas, lo que ha llevado a la necesidad de desarrollar estudios de sensibilidad in vitro que permitan anticipar la eficacia de los antifúngicos, antes de instaurar un tratamiento.

Desde hace unos cinco años se dispone de nuevos compuestos pertenecientes a familias clásicas de antifúngicos (Itraconazol, Voriconazol) de los cuales varios estudios han comenzado a ser difundidos en la literatura médica especializada $(12,16,18,25)$.

La actividad antifúngica es muy variable, dependiendo de los géneros y especies. La Anfotericina B, Caspofungina, Itraconazol y Voriconazol son activos sobre $\boldsymbol{C}$. albicans y Candida no albicans, entre ellas $\boldsymbol{C}$. lusitaniae $(4,25,28,29,30)$.

El Voriconazol presenta un amplio espectro con actividad marcada frente a diferentes especies de Candida spp. por lo que está indicado para el tratamiento de infecciones graves por C. albicans, C. krusei, C. glabrata, C. dubliniensis, C. inconspicua, C. tropicalis, C. guilliermondii y $\boldsymbol{C}$. lusitaniae incluyendo cepas con resistencia intrínseca o adquirida. Tiene una mayor actividad in vitro que sus predecesores, es bien tolerado y tiene efectos adversos mínimos y poco frecuentes. Su uso clínico fue aprobado por la FDA en el año 2002.

Los estudios de sensibilidad in vitro muestran una 
excelente actividad de los azoles de segunda generación frente a Candida spp. incluso en cepas resistentes (16). En un estudio aleatorizado y doble ciego que compara el uso de Voriconazol y Fluconazol en 391 casos de esofagitis candidósica en pacientes inmunodeprimidos se concluye que la eficacia, medida por curación endoscópica, fue similar con una tolerabilidad y seguridad aceptables de ambos fármacos. Los autores concluyen que el Voriconazol resulta eficaz en el tratamiento de esa enfermedad incluso en casos refractarios al Fluconazol (16).

Rubio Calvo et al. (29), sobre 113 aislamientos clínicos de Candida spp. encontraron que el Voriconazol muestra un actividad excelente frente a $\boldsymbol{C}$. lusitaniae y otras Candida spp. Por otra parte, Marco et al.(18), observaron que todas las cepas resistentes al Fluconazol fueron sensibles al Voriconazol. Perfect et al. (23), comunican su experiencia con el tratamiento de rescate con Voriconazol en infecciones fúngicas refractarias o por intolerancia al tratamiento antifúngico indicado previamente. En 87 casos de candidosis invasora (86\%) refractarias obtienen una proporción global de tres pacientes vivos cada cuatro pacientes a los tres meses de iniciado el tratamiento. Estos autores concluyen que el Voriconazol puede tener un impacto positivo en al menos la mitad de los casos de candidosis invasora refractaria. Por último, Ostrosky-Zeichner et al. (21), publicaron recientemente la experiencia del tratamiento de rescate con Voriconazol en 52 casos de candidosis invasora (más del $90 \%$ de enfermedad refractaria al tratamiento antifúngico). La respuesta global fue del $56 \%$. Los autores concluyen que el Voriconazol puede ser un antifúngico adecuado en la terapia de rescate de candidosis invasora incluso en caso de exposición previa a los azoles de primera generación.

En un estudio multicéntrico reciente Hsueh (12), ha comunicado que el Voriconazol fue el agente más efectivo contra C. albicans, $\boldsymbol{C}$. tropicalis, $\boldsymbol{C}$. glabrata, $\boldsymbol{C}$. parapsilosis y $C$. Iusitaniae, incluyendo cepas no susceptibles a Fluconazol ni a Anfotericina B.

En este trabajo describimos el hallazgo de $\boldsymbol{C}$. Iusitaniae en LCR de un paciente pediátrico inmunocomprometido tratado exitosamente con Voriconazol. La cepa resultó resistente a Anfotericina B luego del tratamiento con el fármaco en dosis crecientes durante 12 días. No estamos en condiciones de conocer si se trata de una cepa con resistencia intrínseca o con desarrollo rápido de resistencia al antifúngico.

Consideramos que la presencia cada vez más frecuente de especies resistentes al tratamiento antifúngico, hace necesario la realización rutinaria de pruebas de sensibilidad a toda cepa causante de una candidosis invasiva o que procede de enfermos inmunodeprimidos. Por otra parte, creemos que estos datos de sensibilidad pueden ser muy útiles a la hora de orientar futuros tratamientos empíricos y para valorar epidemiológicamente las resistencias a los antifúngicos según especie y área geográfica.

Por largo tiempo la situación endógena comensal de $\boldsymbol{C}$. lusitaniae se consideró la fuente esencial de contaminación $(5,6)$. Pero tomando en cuenta que los factores iatrogénicos llevan a la selección de cepas oportunistas y a la aparición de especies resistentes a las terapias antifúngicas, obviamente creemos que la especie que hemos aislado tiene su origen en la manipulación de la derivación valvular que se colocó al paciente.

Las expectativas generadas por los nuevos antifúngicos, solos o en combinación, hacen difícil la elección terapéutica y resulta necesario establecer las bases que ayuden a su elección adecuada según tipo de infección y la condición inmunitaria del paciente.

C. Iusitaniae puede ser incorrectamente identificada y confundida con $\boldsymbol{C}$. tropicalis, especie que con mayor frecuencia ha planteado dificultades a la hora de la identificación final. Morfológicamente ambas especies son muy parecidas en los medios de cultivo habituales. Las dos especies producen colonias brillantes de color blanco cremoso, de crecimiento rápido. Microscópicamente presentan blastoconidios a lo largo de las pseudohifas. Pero en el medio CHROM-agar a las 48 h de incubación $\boldsymbol{C}$. Iusitaniae produce colonias de un color que varía entre rosa y púrpura, dependiendo del tiempo de incubación, mientras que $\boldsymbol{C}$. tropicalis produce colonias azul oscuro con un halo púrpura marrón en el agar que la rodea. Por otra parte, el sistema API 32 C contiene los cuatro azúcares (glicerol, ramnosa, gluconato y sorbosa) cuya asimilación resulta clave para la diferenciación entre estas especies. Mientras $\boldsymbol{C}$. lusitaniae asimila estos sustratos azucarados $\boldsymbol{C}$. tropicalis no suele utilizarlos.

\section{AGRADECIMIENTOS}

Al Dr. Silvio Martoni por su asesoramiento y valiosos aportes.

\section{REFERENCIAS}

1. Alexander, B. \& Perfect, J.R . (1997). Antifungal resistance trends towards the year 2000. Drugs 54:667-678

2. Cantón, E.; Viudes, A. \& Pemán, J. (2001). Infección sistémica nosocomial por levaduras. Forum Micológico. Rev. Iberoam. Micol. 18:51-55

3. Chaturvedi, V.; Ramani, R. \& Rex, J. (2004). Collaborative study of antibiotic medium 3 and flow cytometry for identificacion of Amphotericin B-Resistan Candida isolates. J. Clin. Microbiol. 421:2252-54 
4. Favel, A.; Michel-Nguyen,A.; Datry,A.; Challier,S.; Leclerc, F.; Chastin, C. (2004). Susceptibility of clinical isolates of Candida lusitaniae to five systemic antifungal agents. J. Antimicrob. Chemother. 53:536-529

5. Fowler, S.L.; Rhoton, B. \& Pfaller, M. (1998). Evidence for person-to-person transmission of Candida lusitaniae in a neonatal intensive-care unit. Infect. Control Hosp. Epidemiol. $5: 343-345$

6. Fridkin, S. \& Jarvis, W. (1996). Epidemiology of nosocomial fungal infections. Clin. Microbiol. Rev. 9:499-511

7. García-Martos, P.; Domínguez, I.; María, P.; García, R.; Agudo y Mira, J. (2001). Sensibilidad a antifúngicos de levaduras patógenas emergentes. Enf. Infec. y Micobiol. Clínica. 19:249-256

8. Guinet, R.; Chanas, I.; Goullier, A. \& Bonnefoy, G. (1983). Fatal septicemia due to amphotericin-B-resistant Candida lusitaniae. J. Clin. Micorbiol. 18:443-444

9. Haldfield, T. M.; Smith, M.; Winn,R.; Rinaldi, M.G.; Guerra, C. (1987). Mycoses caused by Candida lusitaniae. Rev. Infect. Dis. 9:1006-12

10. Hazen, K.C. (1995). New and emergent yeast pathogens Clin. Microbiol. Reviews 10:462-478

11. Holschu, D. \& Presley, H. (1979). Identification of Candida lusitaniae as an opportunistic yeast in humans. J. Clin. Microbiol. 10:202-205

12. Hsueh, P.; Laus, R. et al. (2005). Antifungal susceptibilities of clinical isolates of Candida species, Cryptococcus neoformans and Aspergillus species from Taiwán: Surveillance of multicenter antimicrobial Resistant. Antimicrob. Agents, Chemother. 49:512517

13. Jarque, I.; Salavert, M.; Romá, E.; Gobernado, M.; Chiveli, M.A.; Ruano, M.; Solé, A. (2004). Guía hospitalaria para la profilaxis y el tratamiento de las infecciones fúngicas en los enfermos inmunodeprimidos o que requieren cuidados especiales. Rev. Esp. Quimioterap. 17:357-389

14. Kutzman , C. \& Fell, J. (1999). The yeasts, a taxonomic study. Elsevier Sci. B.V. $4^{\text {th }}$ Ed.

15. Lewis, R. \& Klepser, M. (1999). The changing face of nosocomial candidemia: epidemiology, resistance and drug therapy. Am. J. Health-Syst. Pharm. 56:525-533

16. Lizasoain, M.; Díaz-Pedroche, C. \& Lumbreras, C. (2004). Aportación de los azoles de segunda generación en las infecciones por Candida spp. Rev. Esp. Quimioterap. 117:105108

17. Lloret Sos, C.; Gutiérrez-Urbón, O. \& Borell-Solé, N. (2002). Candida lusitaniae. http://www.seimc.org/control/ revi_Micol/clusitaniae.htm

18. Marco, F.; Danés,C.; Allmela, C. et al. (2003). Trends in frequency and in vitro susceptibilities to antifungal agents, including voriconazole and anidulafungin, of Candida bloodstream isolates. Result from a six-year study (1996-2001). Diagn. Microbiol. Infect. Dis. 46:259-264

19. Merz, W. (1984). Candida lusitaniae: frequency of recovery, colonization, infection, and amphotericin B resistance. J. Clin. Microbiol. 20:1194-96
20. Nguyen,M.H.; Peacock, J.E.; Morris, A.J.; Tanner, D.C.; Nguyen, M.L.; Snydman, D.R. et al. (1996). The changing face of candidemia: emergence of non-Candida albicans species and antifungal resistance. Am. J. Med. 100:617-23

21. Ostrosky-Zeichner, L.; Oude Lasshoff, M. \& Rex, J.H. (2003). Voriconazole salvage treatment of invasive candidiasis. Eur. J. Clin. Microbiol. Infect. Dis. 22:652-655

22. Pappagianis, D. M.; Collins, R. \& Remington, J. (1979). Development of resistance to amphotericin B in Candida Iusitaniae infecting a human. Antimicrob. Agents Chemother. 16:123-126

23. Perfect, J. R.;Marr,K.; Walsh, T.J. et al. (2003). Voriconazole treatment for less-common emerging or refractory fungal infections. Clin Infect. Diss. 36:1122-1131

24. Peyron, P.; Favel, A.; Michel-Nguyen, A.; Gilly, M.; Regli, P.; Bolstöm, A. (2001). Improved detection of Amphotericin B-resistant isolates of Candida lusitaniae by Etest. J.Clin.Microbiol.Infect.Dis. 20:127-133

25. Pfaller, M. A.; Diekema,D.J.; Messer,S.; Boyken, L.; Hollis, R.; Jones, R. (2003). In vitro activities of Voriconazole, Posaconazole and four licensed Systemic antifungal agents against Candida species infrequently isolated from blood. J. Clin. Microbiol 41:78-83

26. Pietrucha, P.; Dilanchian, R.; Lewis,R.E.; Ahmadd, H.; Lechin, A. (2001). Candida lusitaniae catheter-related sepsis. The Annals Pharmacotherapy 35:1570-1574

27. Rangel-Frausto, M.S. et. al. (1999). National epidemiology of mycoses survey (NEMIS): variation in rates of bloodstream infections due to Candida species in seven surgical intensive care units and six neonatal intensive care units. Clin. Infect. Dis. 29:253258

28. Rex, J. H.; Pfaller,M.A.; Walsh,R.; Chaturvedi, V.; Odds,L.; Rinaldi,M.; Warnock, D. (2001). Antifungal susceptibility testing: Practical aspects and current challenges.Clin. Microbiol. Rev. 14:643-658

29. Rubio Calvo, M.C.; Gil, J. \& Ramírez de Ocáriz, I. (2003). Actividad in vitro de Fluconazol, Voriconazol y Posaconazol frente a Candida spp. Rev. Esp. Quimioterap. 16:227-232

30. Sabo, J. \& Abdel-Rahman, S. (2000). Voriconazole: a new triazole antifungal. Ann. Pharmacother. 34:1032-43

31. Sánchez, V.; Vazquez,J.A.; Barth-Jones, D.; Dembry,L.; Sobewl, J.; Zerve, M.J. (1992) Epidemiology of nosocomial acquisition of Candida lusitaniae. J.Clin.Microbiol.30:3005-08

32. Toscano, C. \& Jarvis, W. (1999). Epidemiology and Clinical aspects of unusual fungal nosocomial infections. Clin. Updates in Fungal Inf. Vol II, Issue I.

33. Yinnon, A. M., K. A. Woodin and K. R. Powell. (1992). Candida lusitaniae infection in the newborn: case report and review of the literature. Pediatr. Infect. Dis. J. 11:878-880

34. Yoon, S. A.; Vazquez,J.; Steffan,P.; Sobel, J.; Akens, R. (1999). High-frequency, in trio reversible switching of Candida lusitaniae clinical isolates from Amphotericin B susceptibility to resistance. Antimicrob. Agents and Chemother. 43:836-845 